\title{
EFEITO DE EXTRATOS DE NIM E DE MÉTODOS DE APLICAÇÃO SOBRE O DANO FOLIAR E O DESENVOLVIMENTO DA LAGARTA-DO-CARTUCHO, Spodoptera frugiperda, EM MILHO
}

\author{
PAULO AFONSO VIANA ${ }^{1}$, HÉLIO TEIXEIRA PRATES ${ }^{1}$ \\ e PAULO EDUARDO DE AQUINO RIBEIRO²
}

\begin{abstract}
${ }^{1}$ Pesquisador da Embrapa Milho e Sorgo. Rod. MG 424, Km 65, Caixa Postal 151, 35701-970, Sete Lagoas, MG, Brasil.E-mail: pviana@cnpms.embrapa.br (autor para correspondência)

${ }^{2}$ Técnico de Nível Superior da Embrapa Milho e Sorgo. Rod. Mg 424. Km 65, Caixa Postal 151, 35701-970, Sete Lagoas, MG, Brasil.
\end{abstract}

Revista Brasileira de Milho e Sorgo, v.6, n.1, p.17-25, 2007

RESUMO - O nim apresenta atividade inseticida e tem perspectiva de uso para o controle de insetos em várias culturas. O objetivo deste trabalho foi avaliar o efeito de extratos de nim e de métodos de aplicação sobre o dano e o desenvolvimento larval de Spodoptera frugiperda em milho. Foram conduzidos dois experimentos em casa-devegetação da Embrapa Milho e Sorgo, em Sete Lagoas, MG, Brasil. No primeiro, avaliaram-se dois extratos de nim e o inseticida chlorpyrifos, com dois tipos de bicos de pulverizador, variando o número de aplicações. No segundo, avaliaram-se três concentrações do extrato aquoso de folhas e o inseticida chlorpyrifos, com duas combinações de bicos, variando o número de aplicações. Os parâmetros avaliados foram o dano foliar causado pela lagarta no milho e o desenvolvimento de lagartas sobreviventes. Os resultados do primeiro experimento mostraram que o menor dano foliar foi obtido com a aplicação do chlorpyrifos $(2,00)$ e com o extrato aquoso de folhas de nim $(2,50)$ aplicado três vezes, com bicos leque. $\mathrm{O}$ extrato comercial apresentou os maiores danos $(7,22 \mathrm{a}$ $7,90)$. O desenvolvimento das lagartas foi consistente com o menor dano nas folhas, resultando no peso de lagarta de $97,41 \mathrm{mg}$. No segundo experimento, os menores danos foliares $(2,62$ e 2,75) foram observados em três aplicações do extrato aquoso, com a conjugação de bicos 80.01/80.04/80.01, utilizando-se as concentrações de 10.000 e 7.500 ppm, respectivamente. $O$ menor peso de lagartas $(60,21 \mathrm{mg})$ foi para a maior concentração do extrato. Conclui-se que o extrato aquoso de folhas de nim (10.000 ppm) aplicado com bicos leque 80.01/80.04/80.01, em três pulverizações, apresenta melhor eficiência no controle de lagartas de $S$. frugiperda em milho. Todas as concentrações avaliadas afetam negativamente o desenvolvimento larval.

Palavras-chave: azadiractina, inseticida natural, Insecta, Zea mays.

\section{EFFECT OF NEEM EXTRACTS AND APPLICATION METHODS ON THE DAMAGE AND DEVELOPMENT OF FALL ARMYWORM, Spodoptera frugiperda, ON MAIZE}

ABSTRACT - The neem tree has a recognized insecticidal activity against insects that damage several crops. The objective of this study was to evaluate the effect of neem exctracts and application methods on the damage and development of Spodoptera 
frugiperda larvae on maize. Two experiments were carried out at Embrapa Milho e Sorgo, Sete Lagoas, MG, Brazil, under greenhouse conditions. Maize leaves were sprayed varying the neem extracts, sprayer nozzles, concentration and number of application. In the first experiment, the results showed the smallest damage to chlorpyrifos (2.00) and for the aqueous extracts from neem leaves (2.50) applied three times through flat nozzles. The commercial extract did not protect maize leaves from larval damage (7.22 to 7.90). The larval development showed consistent with the low leaves damage, resulting in the low larval weight $(97.41 \mathrm{mg})$. In the second experiment, the smallest leaves damage (2.62 and 2.65) were observed in three applications of the aqueous extract, using 80.01/80.04/ 80.01 nozzles, 10,000 and $7,500 \mathrm{ppm}$, respectively. The highest extract concentration provided low larval weight $(60.21 \mathrm{mg})$. In conclusion, the three applications of neem leaves extract $(10,000 \mathrm{ppm})$, using 80.01/80.04/80.01 nozzles, is most effective to protect maize from fall armyworm damage. All the extract concentrations evaluated affect the larval development.

Key words: azadirachtin, natural insecticide, Insecta, Zea mays.

O milho é uma das culturas de maior importância econômica para o Brasil, sendo cultivado em 12,9 milhões de hectares, produzindo cerca de 42,3 milhões de toneladas de grãos (Conab, 2006). Entre os fatores que contribuem para reduzir a produtividade do milho está o complexo de insetos-pragas, destacando-se a lagarta-docartucho, Spodoptera frugiperda (J. E. Smith) (Lepidoptera: Noctuidae). Essa espécie é considerada a principal praga da cultura, pela sua distribuição espacial e temporal, alta incidência em todos os estádios da planta e por causar perdas de até $38,7 \%$ na produção (Williams \& Davis, 1990; Cruz et. al. 1996).

Para o controle dessa praga, geralmente são utilizadas várias aplicações de inseticidas sintéticos, elevando o custo de produção e causando riscos de intoxicação e de contaminação ambiental. Como alternativa a esses inseticidas, destacam-se os inseticidas naturais, que podem ser preparados na própria propriedade e utilizados principalmente por pequenos produtores rurais, contribuindo para reduzir os custos de produção, os riscos e a dependência de inseticidas manufaturados. O nim (Azadirachta indica
A. Juss. Meliaceae) é uma espécie oriunda da Índia e disseminada em outros continentes, apresentando atividade inseticida e com potencial de uso para o controle da lagarta-do-cartucho em milho.

Os derivados do nim têm mostrado acentuada atividade inseticida para várias espécies de pragas (Schumutterer, 1988; Viana \& Prates, 2003). Uma revisão abrangente sobre os efeitos tóxicos do nim para os insetos foi descrita por Mordue \& Nisbet (2000), com ação sobre a alimentação, fisiologia e reprodução. Segundo Schmutterer (1990), os lepidópteros são os mais sensíveis às substâncias oriundas do nim.

A química da planta de nim foi muito estudada na década de 70 e 80 , quando foram identificados mais de 150 compostos (Schmutterer, 1990), sendo os mais ativos pertencentes à classe dos limonóides. A azadiractina é o principal composto produzido pelo nim, com reconhecida atividade inseticida, sendo os frutos a sua principal fonte, além da casca e das folhas (Bruneton, 1995).

A maioria dos derivados da planta estudados para o controle de pragas é obtida por meio 
da moagem das sementes ou da extração de óleo. Blaney et al. (1990) relataram que 1 ppm de azadiractina reduziu a alimentação de Spodoptera littoralis em dieta artificial, quando aplicado sobre um substrato para locomoção da lagarta. Os resultados encontrados por Adel \& Sehnal (2000), para a mesma espécie, mostraram que a mistura de óleo da semente (0,1-10 ppm de azadiractina) na dieta causou interrupção e redução na alimentação, retardamento da ecdise, morte de larvas e pupas e esterilidade dos adultos emergidos. Resultados semelhantes foram obtidos por Prates et al. $(1999,2003)$ e Viana \& Prates (2003) com extrato aquoso de folhas de nim afetando o desenvolvimento e a mortalidade de lagartas recém-eclodidas de S. frugiper$d a$. Viana \& Prates (2005) mostraram que o principal modo de ação do extrato aquoso de folhas de nim é através da ingestão, sendo bastante reduzido o efeito de contato. Isso indica a importância do método de aplicação para uma melhor cobertura foliar visando eficiência no controle dessa praga.

A proteção conferida pelo extrato de nim ao ataque de insetos nas plantas depende de vários fatores. Os bioensaios realizados sob condições controladas de laboratório mostram resultados que necessitam ser ajustados e validados para o uso no campo.

Geralmente, as avaliações de dose-resposta são realizadas com partes de folhas submergidas no extrato de nim, pulverizadas em esteiras ou incorporadas em dietas artificiais, sem qualquer interferência de fatores externos (Torres et al., 2001; Martinez \& Emden, 2001; Viana \& Prates, 2003). O objetivo deste trabalho foi avaliar o efeito de extratos de nim e de métodos de aplicação sobre o desenvolvimento da lagarta-do-cartucho e seu dano causado à planta do milho.

\section{Material e Métodos}

\section{Efeito de extratos de nim e do método de aplicação sobre o controle da lagarta \\ O experimento foi conduzido em casa-} de-vegetação, na Embrapa Milho e Sorgo, em Sete Lagoas, MG. Foram utilizados dois tipos de extrato de nim, sendo um adquirido no mercado (Bioneem) e o outro preparado em laboratório. Para o preparo do extrato, as folhas de nim foram coletadas e secadas ao ar livre e à sombra, durante dez dias. Foram moídas $150 \mathrm{~g}$ (equivalente a $10 \mathrm{~g}$ do extrato bruto) de folhas e colocadas em recipiente contendo $1 \mathrm{~L}$ de água destilada (10 $\mathrm{mg} \mathrm{mL}^{-1}$ ), deixando em repouso por 24 horas. Em seguida, o extrato foi coado em tecido de algodão de malha fina.

Foram semeadas duas sementes de milho em vasos de $5 \mathrm{~L}$. As plantas foram infestadas no estádio de cinco a seis folhas, com dez lagartas recém-eclodidas, e a pulverização foi realizada no dia seguinte. Os tratamentos foram constituídos de dois extratos de nim (comercial e extrato de folhas), um inseticida (chlorpyrifos) e a testemunha (água), dois tipos de bicos do pulverizador (leque e cônico) e duas a três pulverizações (Tabela 2). O chlorpyrifos foi escolhido por ser eficiente no controle da praga e estar há mais tempo no mercado. Cada parcela foi composta por dez vasos com duas plantas. Os tratamentos foram aplicados com um pulverizador costal $\mathrm{CO}_{2}$ (40 lb/pol ${ }^{2}$.) equipado com uma barra tríplice, sendo as pulverizações realizadas com intervalo de dois dias. $O$ delineamento experimental foi o de blocos ao acaso, com duas repetições. Foram avaliados o dano foliar causado pela lagarta utilizando-se uma escala de 0 a 9 (Davis \& Williams, 1989) e o desenvolvimento (biomassa e cápsula cefálica) de lagartas sobreviventes aos 15 dias após a pulverização. 
Os dados avaliados foram submetidos à análise de variância (ANOVA) e as médias comparadas pelo teste Duncan, a 5\% de probabilidade.

\section{Efeito da concentração e do método de aplicação do extrato aquoso de folhas de nim sobre o controle da lagarta}

Esse experimento baseou-se nas informações obtidas no bioensaio anterior e visou reduzir a quantidade de folhas moídas utilizada no preparo da calda do extrato aquoso. No caso, foi testada a variação para menos nas concentrações do extrato e redução do volume da calda através de uso de bico de menor vazão.

A pesquisa foi desenvolvida em casa-devegetação, na Embrapa Milho e Sorgo, em Sete Lagoas, MG. Foram semeadas duas sementes de milho em vasos de $5 \mathrm{~L}$. As plantas foram infestadas no estádio de cinco a seis folhas, com dez lagartas recém-eclodidas, e a pulverização foi realizada no dia seguinte. $\mathrm{O}$ extrato aquoso de folhas de nim usado na pulverização foi preparado utilizando-se a mesma metodologia do experimento anterior. Os tratamentos foram constituídos de três concentrações de extratos aquoso de folhas de nim (10.000, 7.500 e 5.000 ppm), um inseticida (chlorpyrifos) e a testemunha (água), duas combinações de bicos (80.01/80.04/80.01 e $80.01 / 80.03 / 80.01$ ) e duas a três pulverizações (Tabela 3). Cada parcela foi composta por dez vasos com duas plantas. Os tratamentos foram aplicados com um pulverizador costal $\mathrm{CO}_{2}(40$ $\mathrm{lb} / \mathrm{pol}^{2}$.) equipado com uma barra tríplice, sendo as pulverizações realizadas com intervalo de dois dias. O delineamento experimental foi o de blocos ao acaso, com três repetições. Foram avaliados o dano foliar causado pela lagarta, utilizando-se uma escala de 0 a 9 (Davis \& Williams, 1989), e o desenvolvimento (biomassa e cápsula cefálica) de lagartas sobreviventes aos 14 dias após a pulverização.

Os dados avaliados foram submetidos à análise de variância (ANOVA) e as médias, comparadas pelo teste Duncan, a 5\% de probabilidade.

\section{Resultados e Discussão}

\section{Efeito de extratos de nim e do método de aplicação sobre $o$ controle da lagarta}

Os tratamentos avaliados apresentaram diferença significativa $(P<0,005)$ pelo teste $F$ (Tabela 1). O menor dano foliar foi obtido para o chlorpyrifos $(2,00)$, que não diferiu do extrato aquoso de folhas de nim $(2,50)$, aplicado com bicos leque, em três vezes e com intervalo de dois dias. O extrato comercial apresentou os maiores danos $(7,22$ a 7,90) comparando-se ao da testemunha (Tabela 2). Os tipos de bicos e o número de aplicações não contribuíram para melhorar a performance do extrato comercial, não afetando a eficiência de controle de lagarta de $S$. frugiperda no milho, na formulação avaliada.

Para o extrato aquoso, a conjugação de três aplicações utilizando bicos leque (dano de $2,50)$ e bico cônico $(3,95)$ e de duas aplicações com os bicos leques $(4,02)$ foram os tratamentos que apresentaram os menores danos nas folhas (Tabela 2). Para o melhor tratamento com o extrato aquoso, o desenvolvimento das lagartas sobreviventes foi consistente com o dano nas folhas, resultando no segundo menor peso de lagarta $(97,41 \mathrm{mg})$, enquanto as lagartas alimentadas das plantas testemunha atingiram $298,21 \mathrm{mg}$. A largura da cápsula cefálica foi ligeiramente afetada, variando de 2,21 $\mathrm{mm}$ para o melhor tratamento e de 2,84 a $2,89 \mathrm{~mm}$ para a testemunha (Tabela 2). Observou-se que o bico tipo leque associado a três pulverizações foi um fator determinante para se obter uma melhor proteção das 


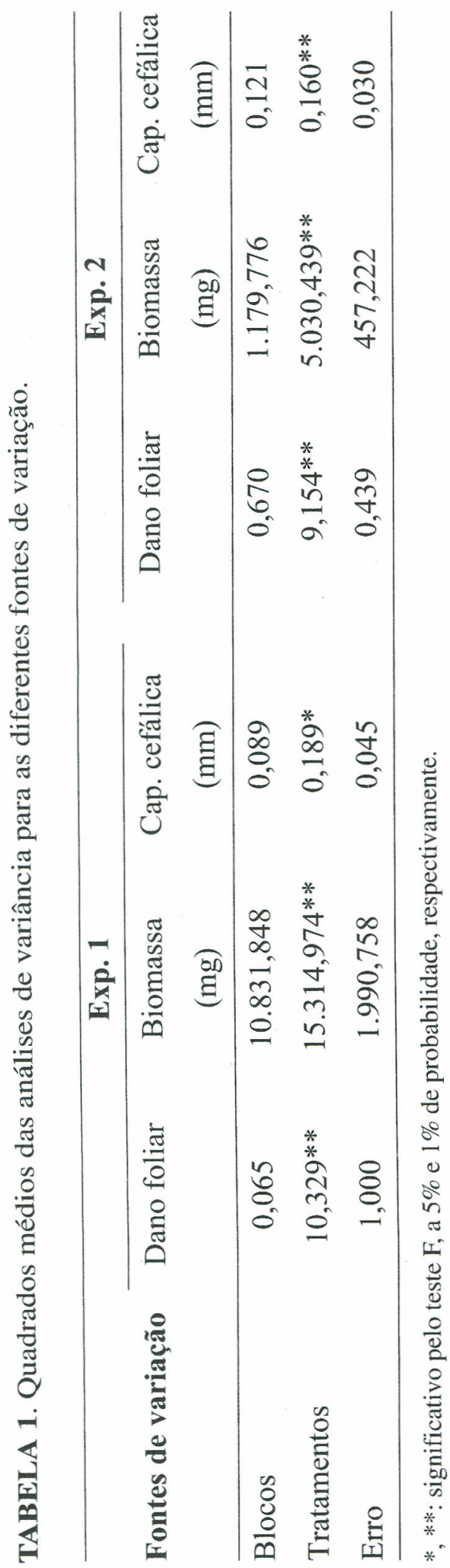

plantas de milho com o extrato aquoso. $\mathrm{O}$ uso de bicos cônicos, mesmo para o chlorpyrifos, que possui efetiva ação de contato e ingestão sobre a lagarta, apresentou o dano relativamente alto $(5,12)$, não sendo suficiente para proteger as plantas do ataque.

Para a lagarta-do-cartucho, o principal modo de ação do extrato aquoso de folhas de nim é por meio da ingestão, sendo bastante reduzido o efeito de contato (Viana \& Prates, 2005). Portanto, ressalta-se a importância da cobertura uniforme do tecido foliar a ser protegido durante a pulverização com o extrato de nim, visando obter melhor eficiência de controle dessa praga. Nas pulverizações realizadas na lavoura do milho, é comum parte das folhas apresentar áreas sem a deposição da calda inseticida. Outro fator que pode contribuir para a redução da eficiência é o rápido crescimento da área foliar e a emergência de novas folhas, possibilitando que as lagartas se alimentem de tecidos foliares não tratados desenvolvidos após a aplicação. Isso indica a importância dos tipos de bicos para a distribuição uniforme da calda sobre as folhas. Os resultados obtidos neste trabalho mostraram que o extrato aquoso pulverizado com bicos leque (80.01/ 80.04/80.01), em três aplicações, visualmente proporcionou a melhor uniformidade de deposição e o menor dano foliar, eqüivalendo ao chlorpyrifos, enquanto, o bico cônico (AD3/AC 13) apresentou baixa eficiência para o controle da praga, tanto para as aplicações dos extratos de nim, quanto para o inseticida químico.

\section{Efeito da concentração e do método de aplicação do extrato aquoso de folhas de nim sobre o controle da lagarta}

Os tratamentos avaliados apresentaram diferença significativa $(P<0,005)$ pelo teste $F$ (Tabela 1). Os menores danos foliares $(2,62 \mathrm{e}$ 


\begin{tabular}{|c|c|c|c|c|c|}
\hline & Tratamentos & & Dano foliar & Biomassa (mg) & Cap. cefálica (mm) \\
\hline Extratos/inseticida & Bicos & $\mathrm{N}^{0}$ de aplicações & & & \\
\hline Extrato aquoso & $80.01 / 80.04 / / 80.01$ & 02 & $4,02 \pm 1,42 b c$ & $127,00 \pm 34,84 a$ & $2,29 \pm 0,19 \mathrm{abc}$ \\
\hline Extrato aquoso & $80.01 / 80.04 / / 80.01$ & 03 & $2,50 \pm 0,25 \mathrm{ab}$ & $97,41 \pm 37,19 \mathrm{a}$ & $2,21 \pm 0,24 \mathrm{ab}$ \\
\hline Extrato aquoso & Disc-core AD3/AC 13 & 02 & $4,95 \pm 0,80 \mathrm{~cd}$ & $163,68 \pm 55,25 b c$ & $2,42 \pm 0,23 \mathrm{abcd}$ \\
\hline Extrato aquoso & Disc-core AD3/AC 13 & 03 & $3,95 \pm 1,05 \mathrm{bc}$ & $77,08 \pm 8,39 a$ & $2,03 \pm 0,11 \mathrm{a}$ \\
\hline Extrato comercial $^{2}$ & $80.01 / 80.04 / / 80.01$ & 02 & $7,67 \pm 0,12 \mathrm{ef}$ & $316,52 \pm 17,41 \mathrm{c}$ & $2,87 \pm 0,07 \mathrm{de}$ \\
\hline Extrato comercial & $80.01 / 80.04 / / 80.01$ & 03 & $7,90 \pm 0,15 \mathrm{ef}$ & $257,33 \pm 18,39 b c$ & $2,76 \pm 0,12 \mathrm{cde}$ \\
\hline Extrato comercial & Disc-core AD3/AC 13 & 02 & $7,22 \pm 0,87 \mathrm{def}$ & $176,32 \pm 54,74 b c$ & $2,55 \pm 0,28 \mathrm{bcde}$ \\
\hline Extrato comercial & Disc-core AD3/AC 13 & 03 & $7,85 \pm 0,50 \mathrm{ef}$ & $297,15 \pm 54,08 \mathrm{c}$ & $2,83 \pm 0,08 \mathrm{de}$ \\
\hline Chlorpyrifos $^{3}$ & $80.01 / 80.04 / / 80.01$ & 01 & $2,00 \pm 0,15 a$ & $302,85 \pm 42,55 \mathrm{c}$ & $2,95 \pm 0,15 \mathrm{e}$ \\
\hline Chlorpyrifos & Disc-core AD3/AC 13 & 01 & $5,12 \pm 0,72 \mathrm{cde}$ & $240,53 \pm 4,01 b c$ & $2,69 \pm 0,04$ bcde \\
\hline Test. (água) & $80.01 / 80.04 / / 80.01$ & 01 & $8,07 \pm 0,07 \mathrm{f}$ & $290,62 \pm 47,13 c$ & $2,89 \pm 0,06 \mathrm{de}$ \\
\hline Test. (água) & Disc-core AD3/AC 13 & 01 & $7,90 \pm 0,30 \mathrm{ef}$ & $298,21 \pm 7,51 \mathrm{c}$ & $2,84 \pm 0,01 \mathrm{de}$ \\
\hline \multicolumn{6}{|c|}{$\begin{array}{l}\text { 'Médias ( } \pm \text { EP) seguidas da mesma letra não diferem significativamente pelo teste de Duncan a } 5 \% \text { de probabilidade. } \\
\text { }{ }^{2} \text { Bioneem ( } 1 \text { L/100 L de água). } \\
{ }^{2} 288 \text { g i.a./ha. }\end{array}$} \\
\hline
\end{tabular}


2,75) foram observados em três aplicações do extrato com a conjugação de bicos leque $80.01 /$ 80.04/80.01, utilizando-se as concentrações de 10.000 e 7.500 ppm. A aplicação de chlorpyrifos e a testemunha apresentaram dano médio de 0,48 e 7,83, respectivamente (Tabela 3). Em geral, os tratamentos com os extratos aquoso de nim reduziram o desenvolvimento larval. O peso de lagartas variou de 60,21 a 100,8 mg e a largura da cápsula cefálica variou de 1,77 a $2,10 \mathrm{~mm}$, enquanto, na testemunha, o peso foi de $216,30 \mathrm{mg}$ e a cápsula cefálica de 2,67 mm (Tabela 3).

Segundo Schmutterer (1988) e Mordue \& Blackwell (1993), a morte dos insetos-alvos tratados com o nim depende da dose e do tempo de exposição ao princípio ativo. Resultados obtidos para a $S$. frugiperda com o extrato aquoso de folhas de nim incorporado na dieta, nas concentrações de 10.000 e $3.600 \mu \mathrm{g} . \mathrm{mL}^{-1}$, proporcionaram eficiência de controle de 100,0 e 79,4\%, respectivamente (Prates et al., 2003). No entanto, para se avaliar a eficácia no controle dessa praga, em campo, são necessários ajustes para se obter uma eficiência equivalente aos ensaios conduzidos em laboratório. Isso foi confirmado pelos resultados, os quais indicaram que a menor concentração utilizada proporcionou, em laboratório, eficiência acima de 79,4\%, mas quando pulverizado sobre a planta de milho, não controlou eficientemente a lagarta, resultando em danos que variaram de 4,18 a 5,85 (Tabela 3 ), podendo ocasionar perdas em produtividade.

Outro aspecto a considerar ao utilizar extratos de nim é que os insetos são capazes de

TABELA 3. Efeito da concentração e do método de aplicação do extrato aquoso de folhas de nim sobre o controle de lagartas de Spodoptera frugiperda ${ }^{1}$.

\begin{tabular}{|c|c|c|c|c|c|}
\hline \multicolumn{3}{|c|}{ Tratamentos } & \multirow[t]{2}{*}{ Dano foliar } & \multirow{2}{*}{$\begin{array}{c}\text { Biomassa } \\
\text { (mg) }\end{array}$} & \multirow{2}{*}{$\begin{array}{c}\text { Cap. cefálica } \\
\text { (mm) }\end{array}$} \\
\hline Concentração (ppm) & Bicos & $\mathrm{N}^{0}$ de aplicações & & & \\
\hline 10.000 & $80.01 / 80.04 / 80.01$ & 02 & $3,78 \pm 0,25$ defg & $60,21 \pm 9,60 b c$ & $1,94 \pm 0,05 b c$ \\
\hline 10.000 & $80.01 / 80.04 / 80.01$ & 03 & $2,62 \pm 0,21 \mathrm{~g}$ & $60,34 \pm 14,86 b c$ & $1,82 \pm 0,14 b c$ \\
\hline 10.000 & $80.01 / 80.03 / 80.01$ & 02 & $4,80 \pm 0,56 b c d$ & $72,47 \pm 5,06 \mathrm{bc}$ & $1,92 \pm 0,02 b c$ \\
\hline 10.000 & $80.01 / 80.03 / 80.01$ & 03 & $3,15 \pm 0,05$ efg & $62,28 \pm 1,58 b \mathrm{c}$ & $1,88 \pm 0,05 b c$ \\
\hline 7.500 & $80.01 / 80.04 / 80.01$ & 02 & $5,07 \pm 0,24 b c$ & $100,80 \pm 6,97 b$ & $2,00 \pm 0,03 b c$ \\
\hline 7.500 & $80.01 / 80.04 / 80.01$ & 03 & $2,75 \pm 0,45 \mathrm{fg}$ & $63,51 \pm 11,26 \mathrm{bc}$ & $1,77 \pm 0,14 b c$ \\
\hline 7.500 & $80.01 / 80.03 / 80.01$ & 02 & $3,90 \pm 0,66 \mathrm{cdef}$ & $78,78 \pm 5,54 b c$ & $1,99 \pm 0,10 \mathrm{bc}$ \\
\hline 7.500 & $80.01 / 80.03 / 80.01$ & 03 & $3,63 \pm 0,13$ defg & $79,88 \pm 10,13 b c$ & $1,90 \pm 0,13 b c$ \\
\hline 5.000 & $80.01 / 80.04 / 80.01$ & 02 & $5,80 \pm 0,43 b$ & $92,55 \pm 8,50 b$ & $2,05 \pm 0,04 \mathrm{~b}$ \\
\hline 5.000 & $80.01 / 80.04 / 80.01$ & 03 & $4,22 \pm 0,58 \mathrm{cde}$ & $73,30 \pm 18,49 b c$ & $1,91 \pm 0,11 b c$ \\
\hline 5.000 & $80.01 / 80.03 / 80.01$ & 02 & $4,18 \pm 0,17 \mathrm{cde}$ & $94,12 \pm 20,71 b$ & $2,10 \pm 0,14 b$ \\
\hline 5.000 & $80.01 / 80.03 / 80.01$ & 03 & $5,85 \pm 0,61 b$ & $75,33 \pm 13,88 b c$ & $1,93 \pm 0,04 b c$ \\
\hline chlorpyrifos & $80.01 / 80.04 / 80.01$ & 01 & $0,48 \pm 0,17 \mathrm{~h}$ & $45,96 \pm 21,10 \mathrm{c}$ & $1,69 \pm 0,24 \mathrm{c}$ \\
\hline Test. (água) & $80.01 / 80.04 / 80.01$ & 01 & $7,83 \pm 0,20 \mathrm{a}$ & $216,30 \pm 15,61 \mathrm{a}$ & $2,68 \pm 0,04 a$ \\
\hline
\end{tabular}

${ }^{1}$ Médias ( \pm EP) seguidas da mesma letra não diferem significativamente pelo teste de Duncan a $5 \%$ de probabilidade. 
diferenciar as partes tratadas e não tratadas (Schmutterer, 1990), mostrando que a pulverização deve ser cuidadosamente realizada em alto volume e usando técnicas que garantam a distribuição uniforme da calda. Entretanto, mudanças no volume da calda, proporcionada pela troca de bicos 80.01/80.04/80.01 para 80.01/80.03/80.01 e redução na concentração de 10.000 para 7.500 $\mathrm{ppm}$, tiveram pouco efeito na proteção contra o dano foliar causado pela lagarta, quando mantidas três aplicações do extrato. Essa possibilidade de ajustes, principalmente na concentração do extrato, foi relatada por Prates et al. (2003). Porém, a redução no número de aplicações foi favorável ao desenvolvimento das lagartas e, conseqüentemente, aumentou o dano foliar.

Os tratamentos com o extrato aquoso de folhas de nim mais concentrados mostraram melhor proteção ao se utilizar três aplicações. Esse resultado está de acordo com o relatado por Schmutterer (1990), em que múltiplas aplicações do extrato geralmente conferem melhor proteção da planta ao ataque de pragas. Isso pode ser atribuído ao crescimento dinâmico da planta e ao surgimento de novos tecidos foliares, que serão melhor protegidos pelo maior número de aplicações. Manter a área foliar coberta uniformemente pelo extrato obriga a lagarta a alimentar-se dos tecidos tratados, pois um dia de alimentação é tempo suficiente para causar alta mortalidade (Viana \& Prates, 2005). Além disso, múltiplas aplicações podem ser necessárias, devido à sensibilidade da azadiractina à fotodegradação, que contribui para reduzir a ação inseticida pelos raios ultra-violeta (Johnson et al., 2003).

\section{Conclusões}

O extrato aquoso de folhas de nim ( $10.000 \mathrm{ppm}$ ), aplicado com bicos leque $80.01 /$ $80.04 / 80.01$, em três pulverizações, apresenta melhor eficiência no controle de lagartas de $S$. frugiperda em milho.

Bico cônico (AD3/AC 13) proporciona baixa eficiência para a aplicação de extratos de nim para o controle de lagartas de $S$. frugiperda em milho.

$\mathrm{O}$ extrato aquoso de folhas de nim afeta negativamente o desenvolvimento larval de $S$. frugiperda.

\section{Literatura Citada}

ADEL, M. M.; SEHNAL, F. Azadirachtin potentiates the action of ecdsysteroid agonist RH-2485 in Spodoptera littoralis. Journal of Insect Physiology, Oxford, v. 46, p. 267-274, 2000.

BLANEY, W. M.; SIMMONDS, M. S. J.; LEY, W. V.; ANDERSON, J. C.; TOOGOOD, P. L. Antifeedant effects of azadirachtin and structurally related compounds on lepidopterous larvae. Entomologia Experimentalis et Applicata, Dordrecht, v. 55, p. 149-160, 1990.

BRUNETON, J. Pharmacognosy, phytochemistry, medicinal plants. Andover: Intercept; $\mathrm{Pa}$ ris: Lavoisier, 1995. 915 p.

CRUZ, I.; OLIVEIRA, L. J.; OLIVEIRA, A. C.; VASCONCELOS, C. A. Efeito do nível de saturação de alumínio em solo ácido sobre os danos de Spodoptera frugiperda (J. E. Smith) em milho. Anais da Sociedade Entomológica do Brasil, Londrina, v. 25 , n. 2, p. 293-297, 1996.

DAVIS, F. M.; WILLIAMS, W. P. Methods used to screen maize for and to determine mechanisms of resistance to the Southwestern corn borer and Fall armyworm. In: INTERNATIONAL SYMPOSIUM ON METHODOLOGIES FOR 
DEVELOPMENT HOST PLANT RESISTANCE TO MAIZE INSECTS, 1989, México. Proceedings... Mexico: CIMMYT, 1989. p. 101-104

JOHNSON, S.; DUREJA, P.; DHINGRA, S. Photostabilizers for azadirachtin-A (a neem-based pesticide). Journal of Environmental Science and Health, New York, v. b38, n. 4, p. 451462, 2003.

MARTINEZ, S. M.; EMDEN, H. F. van. Growth disruption, abnormalities and mortality of Spodoptera littoralis (Baisduval)(Lepidoptera: Noctuidae) caused by azadirachtin. Neotropical Entomology, Londrina, v. 30, n.1, p. 113125, 2001.

MORDUE, A. J.; BLACKWELL, A. Azadirachtin: An Update. Journal of Insect Physiology, Oxford, v. 39, p. 903-924, 1993.

MORDUE, A. J.; NISBET, A. Azadirachtin from the neem tree Azadirachta indica: its actions against insects. Anais da Sociedade Entomológica do Brasil, Londrina, v. 29, p. 615-632, 2000.

PRATES, H. T.; VIANA, P. A. Determinação de concentrações de extratos de Nim (Azadirachta indica) para o controle da lagarta-docartucho (Spodoptera frugiperda), em laboratório. Sete Lagoas: EMBRAPA-CNPMS, 1999. 4 p. (EMBRAPA-CNPMS.Pesquisa em Andamento, 39).

PRATES, H. T.; VIANA, P. A.; WAQUIL, J. M. Atividade de Extrato Aquoso de Nim (Azadirachta indica A. Juss) sobre Spodoptera frugiper- $d a$ (J. E. Smith). Pesquisa Agropecuária Brasileira. Brasília, DF:, v. 38, n. 3, p. 437-439, 2003.

SCHMUTTERER, H. Potential of azadirachtincontaining pesticides for integrated pest control in developing and industrialized countries. Journal of Insect Physiology, Oxford, v. 34, p. 713$719,1988$.

SCHUMUTTERER, H. Properties and potential of natural pesticides from the neem tree, Azadirachta indica. Annual Review of Entomology, Palo Alto, v. 35, p. 271-297, 1990.

TORRES, A. L.; BARROS, R.; OLIVEIRA, J. V. de. Efeito de extratos aquosos de plantas no desenvolvimento de Plutella xylostella (L.)(Lepidoptera: Plutellidae). Neotropical Entomology, Londrina, v. 30, n. 1, p. 151-156, 2001.

VIANA, P. A.; PRATES, H. T. Desenvolvimento e mortalidade larval de Spodoptera frugiper$d a$ em folhas de milho tratadas com extrato aquoso de folhas de Azadirachta indica. Bragantia, Campinas, v. 62, p. 69-74, 2003.

VIANA, P. A.; PRATES, H. T. Mortalidade de lagarta de Spodoptera frugiperda alimentadas com folhas de milho tratadas com extrato aquoso de folhas de nim (Azadirachta indica). Revista Brasileira de Milho e Sorgo, Sete Lagoas, v. 4, n.. 3, p. 316-322, 2005.

WILLIAMS, W. P.; DAVIS, F. M. Response of corn to artificial infestation with fall armyworm and southwestern corn borer larvae. Southwestern Entomologist, Dallas, v.15, p.163-166, 1990. 